\title{
Local Residual Stress Analysis on Deep Drawn Cups by Means of the Incremental Hole-Drilling Method
}

\author{
Simone Schuster ${ }^{1, a^{*}}$, Jan Pagenkopf ${ }^{1,2, b}$ and Jens Gibmeier ${ }^{1, c}$ \\ ${ }^{1}$ Karlsruhe Institute of Technology (KIT), Institute for Applied Materials (IAM), Kaiserstr. 12, \\ D-76131 Karlsruhe, Germany \\ ${ }^{2}$ Fraunhofer Institute for Mechanics of Materials IWM, Wöhlerstr. 11, D-79108 Freiburg, \\ Germany \\ a simone.schuster@kit.edu, ${ }^{b}$ jan.pagenkopf@iwm.fraunhofer.de, ${ }^{c}$ jens.gibmeier@kit.edu
}

\begin{abstract}
Keywords: Incremental Hole-drilling Method, Crystallographic Texture, Multiple CaseSpecific Calibration Functions, Deep Drawing
\end{abstract}

\begin{abstract}
In addition to residual stresses sheet metal forming induces characteristic crystallographic texture, hence, the material behavior is anisotropic. In general, the standard evaluation procedures of residual stress analysis techniques are limited to isotropic material states. In the present paper deep drawn steel cups of dual phase steel DP600 are analyzed by using a recently proposed calibration approach for residual stress analysis by means of the incremental hole-drilling method for highly textured material states. It is based on the differential method, which is enhanced with four case specific calibration functions. The multiple case specific calibration functions are determined by means of finite element simulations using the orientation distribution function (ODF) in combination with Hill's assumption and single crystal elastic constants of iron to calculate the effective elasticity tensor to account for elastic anisotropy. Supplementary, the deep drawing process is simulated using a finite element model based on the Hill48 yield criterion. Finally, the comparison shows that the numerical results are in satisfactory agreement to the experimental data.
\end{abstract}

\section{Introduction}

Standard methods of residual stress measurement techniques are restricted to isotropic material states. However, forming processes like e.g. rolling or deep drawing cause preferred orientations of the grains due to the limited possibilities of gliding. Crystallographic textures oftentimes result in anisotropic material behavior.

The standard approach of X-ray diffraction stress analysis according to the $\sin ^{2} \psi$-method [1] is no longer applicable in case of textured material states, since the $2 \theta-\sin ^{2} \psi$ distributions are strongly nonlinear. A remedy is the application of special measurement strategies like e.g. the crystallite group method [2] and stress factors [3], where the texture of the sample must be known a priori. These measurement strategies are elaborate and time-consuming and the knowledge of the stress free lattice parameter $\mathrm{D}_{0}$ is required. Furthermore, formed components often obtain complex geometries and can be large (e.g. A-/B-/C-pillar, cowl). Shadowing effects can occur due to the complex shape of the sample. Owing to a limited installation space of the X-ray diffractometer the samples have to be cut and stress redistributions must be considered during the residual stress calculation. Since the penetration depth of conventionally generated X-rays is limited to a few microns, layers of the material must be removed by means of e.g. electrochemical polishing to determine residual stress depth distributions.

The incremental hole-drilling method has great potential, since it is versatile and fast compared to X-ray diffraction. Standard stress calculation methods (e.g. integral method [4], differential method [5]) use calibration data, which is based on isotropic material behavior. Significant errors in stress calculation can occur, if conventional calibration data is applied to residual stress analysis on 
strongly textured materials [6]. Thus, we proposed a new calibration approach based on the differential method [7]. Four case-specific calibration functions must be determined numerically to account for the anisotropic elastic material properties. The elastic constants of the textured sample must be known beforehand for the calibration. A model assumption considering the interactions of the grain boundaries e.g. Voigt, Reuss, Eshelby/Kröner or Hill [8] can be used to calculate the effective elasticity tensor by means of the orientation distribution function (ODF) and single crystal elastic constants. In the present study residual stress depth distributions are determined at different positions of a deep drawn cylindrical cup made of dual phase steel DP600. They are compared to results obtained from finite element (FE) simulations of the deep drawing process.

\section{Experimental investigation}

A deep drawn steel cup with a nominal diameter of $100 \mathrm{~mm}$ and a drawing ratio of 1.8 , which is the relationship between the blank diameter prior to the drawing operation and the punch diameter, was analyzed. Here, a cold rolled dual phase steel sheet DP600 (ferritic-pearlitic microstructure, model sample taken out during the rolling process prior to the final heat treatment) with a thickness of $1 \mathrm{~mm}$ was deep drawn with a blank holder force of $180 \mathrm{kN}$. The deep drawing operation leads to characteristic earing at the edge of the cup caused by the planar anisotropy of the material. Three different measuring locations were defined as can be seen in Fig. 1. A measuring location below an ear (path I) and one below a trough (path II) are located at the half of the maximum cup height. The rolling direction (RD) of the steel sheet corresponds to path II. The third measuring position is located in RD at the radius between cup wall and bottom. Stress components in drawing direction (DD) and tangential direction (TD) were determined. The chemical composition of the dual phase steel is shown in Table 1.

Table 1: Chemical composition of DP600 in weight-\%

\begin{tabular}{|c|c|c|c|c|c|c|c|c|c|}
\hline $\mathrm{C}$ & $\mathrm{Si}$ & $\mathrm{Mn}$ & $\mathrm{P}$ & $\mathrm{S}$ & $\mathrm{B}$ & $\mathrm{Al}$ & $\mathrm{Cr}$ & $\mathrm{Mo}$ & $\mathrm{Ni}$ \\
\hline 0.093 & 0.29 & 1.663 & 0.011 & $<0.001$ & 0.0002 & 0.042 & 0.341 & 0.007 & 0.047 \\
\hline
\end{tabular}
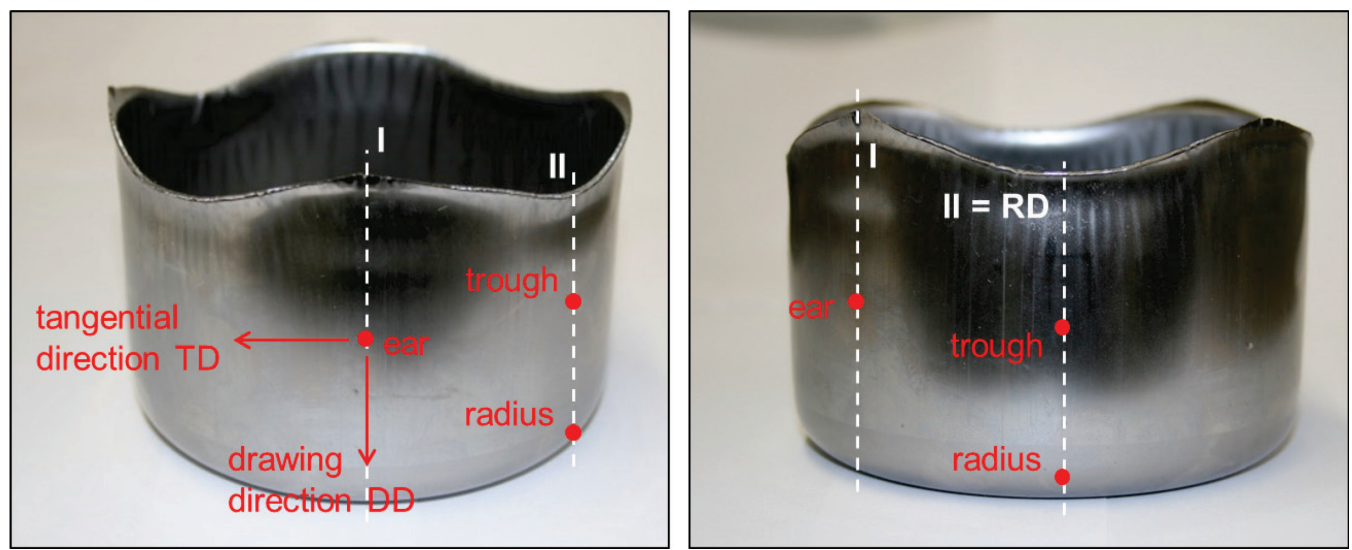

Fig. 1: Definition of measuring locations 'ear', 'trough' and 'radius' and directions.

X-ray diffraction texture analyses were carried out using a diffractometer of type XRD 3003 PTS from Seifert. A pin hole collimator with a nominal diameter of $1 \mathrm{~mm}$ and $\mathrm{CoK} \alpha$ radiation was used. Incomplete pole figures for the lattice planes of type $\{110\},\{200\},\{211\}$ and $\{220\}$ were measured using a $\beta$-range of $0^{\circ} . .65^{\circ}$ and a $\alpha$-range of $-170^{\circ} . .170^{\circ}$ each in a step size of $5^{\circ}$. Fig. 2 illustrates the pole figures $\{100\},\{110\}$ and $\{111\}$, which were recalculated from the ODF. The rolling direction of the cold rolled steel sheet prior to the deep drawing operation is still visible for the 'trough' and 'radius' location and points into the drawing direction. In contrast, the maximum intensity in the pole figures at the measuring position below the ear is turned $45^{\circ}$ with respect to the drawing direction. 
The ODF, Hill's model assumption [8] and the single crystal elastic constants of iron $\left(\mathrm{C}_{11}=230 \mathrm{GPa}\right.$, $\mathrm{C}_{12}=135 \mathrm{GPa}$ and $\mathrm{C}_{44}=117 \mathrm{GPa}$ [9]) were used to determine the elasticity tensor $\mathrm{C}_{\mathrm{ijk} \mathrm{kl}}$. The crystallographic texture leads to a Young's modulus ratio $E_{\max } / E_{\min }$ within the measuring plane of 1.09 in case of the trough and the radius and 1.03 in case of the ear.
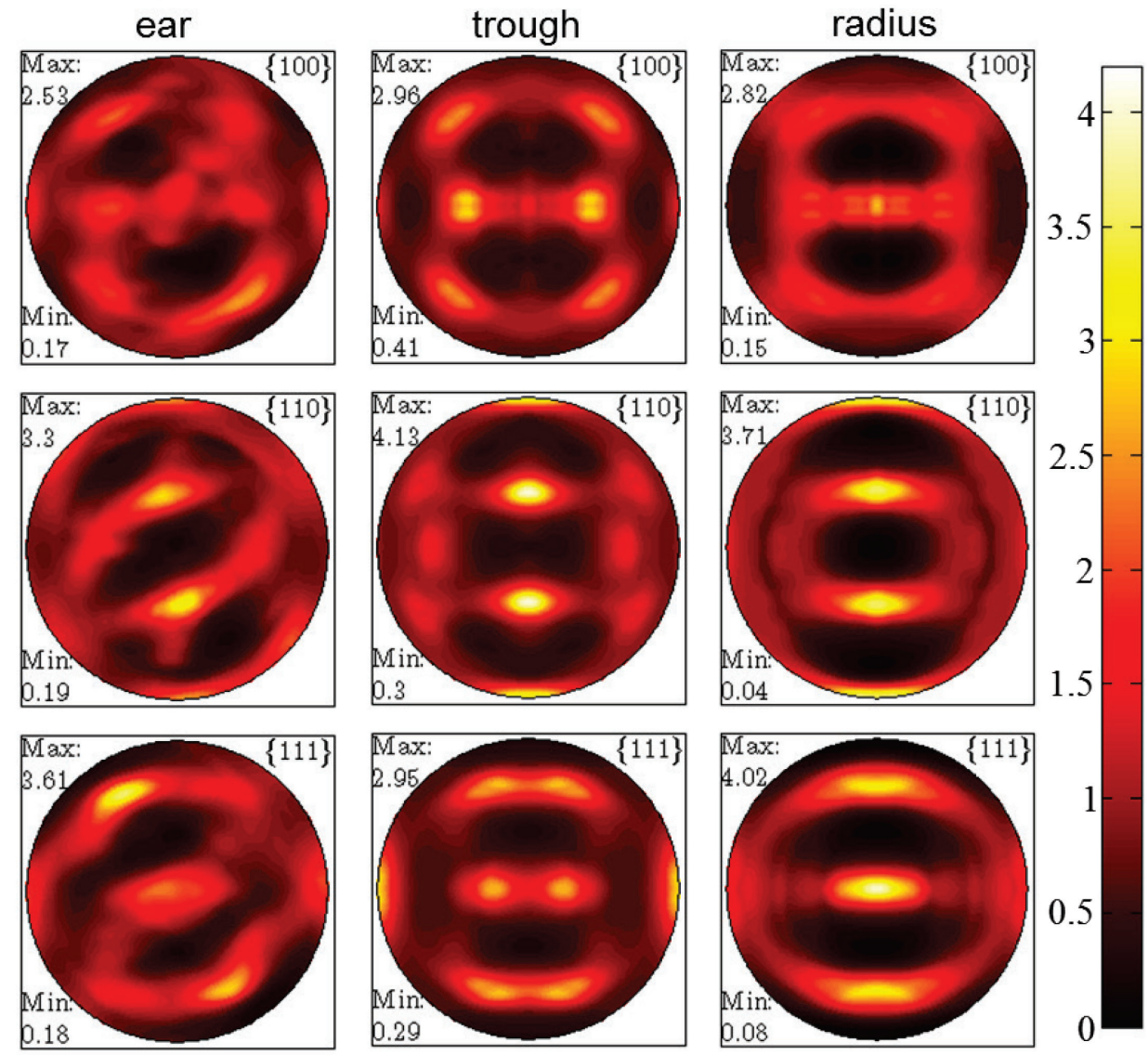

Fig. 2: Pole figures of type $\{100\},\{110\}$ and $\{111\}$ for the three measuring locations 'ear', 'trough' and 'radius'.

A hole-drilling device RS200 from Vishay Measurement Group was used for the hole-drilling experiments. TiN coated end mills with a nominal diameter of $0.8 \mathrm{~mm}$ were used. Accordingly, strain gage rosettes of type EA-11-031RE-120 from Vishay Measurement Group were applied on the sample. The residual stress depth profiles were calculated using the new calibration approach based on the differential method and multiple case-specific calibration functions considering the elastic anisotropy, which we recently proposed in [7]. The following steps have to be conducted:

(i) Texture measurement and determination of the ODF

(ii) Calculation of the elasticity tensor $C_{\mathrm{ijkl}}$

(iii) Determination of four case-specific calibration functions (two FE simulations required)

(iv) Residual stress calculation

Basically, the residual stress components in the two perpendicular directions of the rosette can be determined with this approach. The FE model shown in [7] was used for the determination of the case-specific calibration functions. The elastic anisotropy was considered by means of the elasticity tensor. Additionally, the FE model accounts for the small component's thickness. An external load was applied to the outside surfaces of the model to induce an in-plane calibration stress. Furthermore, the direction of calibration stress was aligned to the orientation of the elastic constants in drawing 
and tangential direction of the cup. Drilling of the hole was realized by a stepwise deletion of the elements. An integral strain relaxation over the strain gage area was calculated for each drilling step.

Complementary, XRD stress analyses by means of the $\sin ^{2} \psi$-method were carried out. Due to the limited installation space of the stationary diffractometer the sample must be cut, which causes complex redistributions of the original residual stresses. Furthermore, non-linear $2 \theta-\sin ^{2} \psi$ distributions were obtained. Thus, by this means no reliable determination of the residual stress state on the analyzed cup was suitable.

\section{Finite element simulation of the deep drawing process}

The numerical simulation of the deep drawing process was performed using the finite element software package Abaqus. First, the forming of the cylindrical cup was simulated with an explicit time integration scheme. An elasto-plastic model with isotropic hardening was used to describe the material behavior. The comparison between the punch forces measured during the forming process and predicted by the simulation was used to adjust the flow curve of the material for high strains. In order to account for the plastic anisotropy of the sheet, the Hill48 [10] yield function was applied. The anisotropy parameters were determined to give the best fit to both the Lankford parameters (rvalues) and yield stresses evaluated from tensile tests in three directions $\left(0^{\circ}, 45^{\circ}\right.$ and $90^{\circ}$ to rolling direction). Due to the material and specimen symmetry, only one quarter of the blank was modeled. The sheet was meshed with brick elements with reduced integration (C3D8R) using six elements over the sheet thickness. The tools were modeled as analytical rigid bodies and the friction coefficient was set to $\mu=0.09$ for all contact pairs. After the forming step, an elastic springback simulation was performed using the implicit time integration scheme of Abaqus/Standard to obtain the residual stresses in the final component.

\section{Results and discussion}

Figure 3 shows the numerically determined residual stress distribution for the components in drawing (a) and tangential (b) direction of the deep drawn cup. The measuring locations are marked by black dots. The FE model is appropriate to describe the characteristic earing of the cup. Steep gradients for the stress in drawing direction can be detected on the outside surface of the cup. An area with maximum tensile stresses of more than $1000 \mathrm{MPa}$ is located approx. $15 \mathrm{~mm}$ below the trough (marked by a black X). Actually, two belts of high tensile stresses ( $>500 \mathrm{MPa}$ ) along the outer surface can be observed. In contrast, the stress distribution of the component in tangential direction (3b) shows lower lateral gradients. Only at the edges of the cup very high tangential stresses (approx. $1000 \mathrm{MPa}$ ) can be detected. Again, an area with high tensile stresses of approx. $500 \mathrm{MPa}$ is located below the trough. A comparison of the numerically and experimentally determined stress depth distributions for the three measuring locations is illustrated in Fig. 4. The hole-drilling experiments were evaluated up to a drilling depth of $0.4 \mathrm{~mm}$. It is worth noting that the wall thickness of the cup is approx. $1 \mathrm{~mm}$. The measurement accuracy of the incremental hole-drilling method is $\pm 25 \mathrm{MPa}$ for standard applications. It is slightly higher in case of textured material states due to the higher experimental effort. In general, a good qualitative and quantitative agreement between the numerical and experimental results was achieved as indicated by the plots in Fig. 4. At the measuring location 'ear' the residual stresses in drawing and tangential direction are almost identical and constant over depth. The numerically determined residual stress profile in tangential direction is in very good accordance to the experimental result. For the stress component in drawing direction a gap with an average deviation of approx. $67 \mathrm{MPa}$ exist. In case of the measuring location 'trough' the residual stress in drawing direction is slightly overestimated by the FE model. Whereas, the difference of the stress component in tangential direction is higher (approx. $218 \mathrm{MPa}$ ) especially near to the surface. The tensile residual stress distributions at the measuring location 'radius' are lowest of all measuring positions. The numerical results are conforming with the experimental results with an accuracy better than $60 \mathrm{MPa}$. 
a) drawing direction

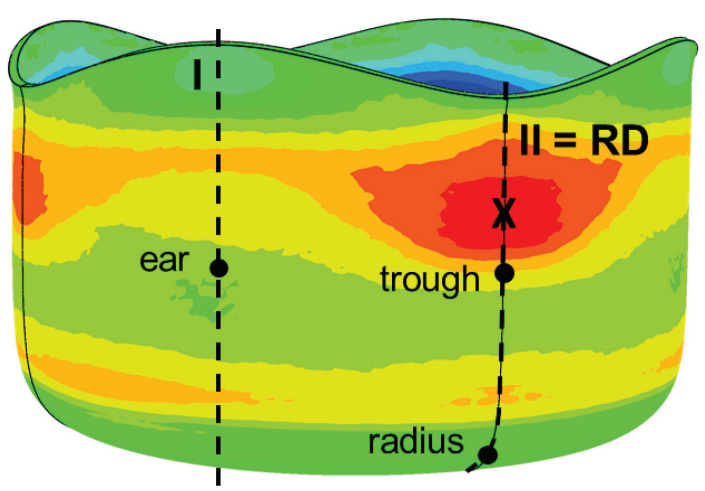

b) tangential direction

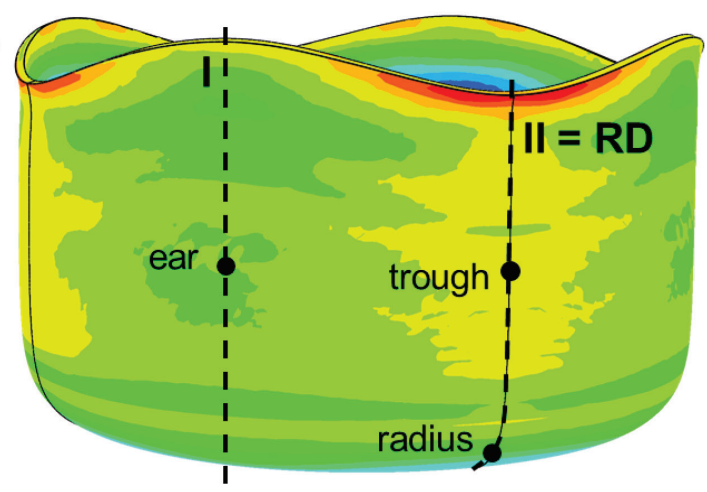

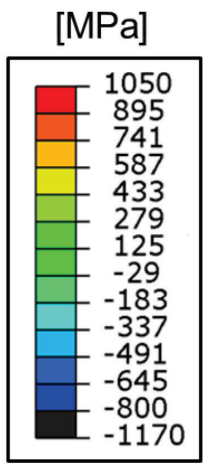

Fig. 3: Distribution of the stress components in drawing (a) and tangential direction (b) calculated by means of FE simulation.
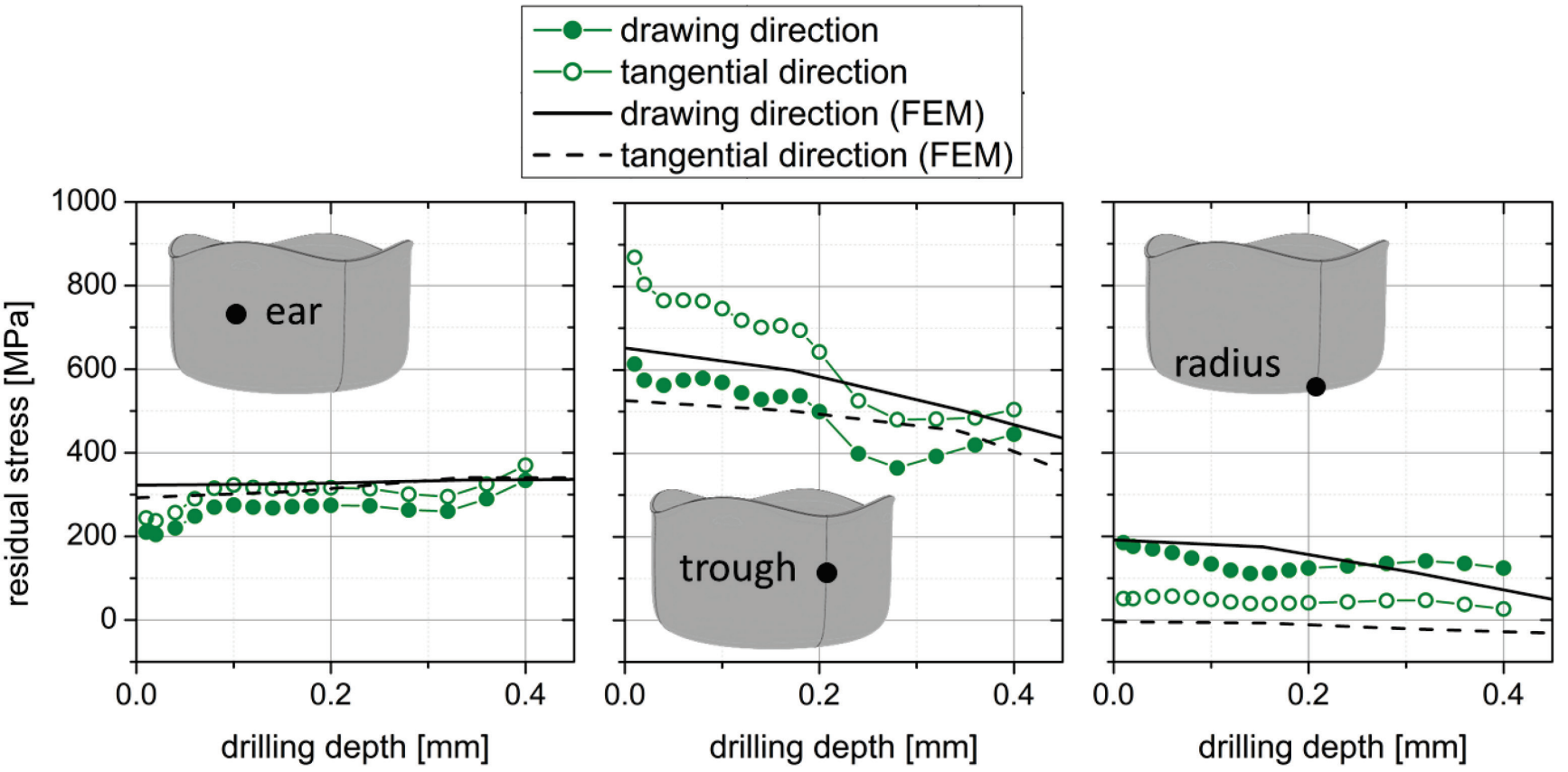

Fig. 4: Comparison of residual stress depth distributions between experimental and numerical results.

Despite the large lateral residual stress gradients especially for the measuring location 'trough' (as indicated by Fig. 3) a good agreement between experiment and FE model could be achieved. Hence, with recourse to the preliminary studies in $[6,7]$ we can conclude that the new calibration approach should always be applied in case of textured material states regardless of the additional effort. Deviations between experiment and FE model can be explained by (i) the simplifying assumptions in the FE model (no kinematic hardening, lack of data of the flow curve for high strains), (ii) large residual stress gradients near the measuring locations and (iii) high residual stresses with respect to the materials yield strength (i.e. measuring position 'trough'), which can cause plastic deformations in the vicinity of the drilled hole and thus, an overestimation of the residual stress magnitude.

\section{Conclusion}

We demonstrated a reliable residual stress analysis by means of the incremental hole-drilling method on a deep drawn cup with a strong local anisotropy using an evaluation approach based on multiple case-specific calibration functions, which we proposed in [7]. The knowledge of the effective 
elasticity tensor $\mathrm{C}_{\mathrm{ijkl}}$ is required. It can be calculated using the ODF (texture measurement) in combination with single crystal elastic constants of the material and a model assumption of e.g. Hill [8]. The case-specific calibration functions can also account for the component's thickness. Since the wall thickness of the cup is thinner than the application limit of the incremental hole-drilling method, it is highly recommended to consider the component's thickness for the provision of the calibration data. The deep drawing operation was simulated using a FE model. Although, we had no experimental data for the yield behavior for large deformations, the numerical results are in satisfactory accordance to the experimental residual stress depth distributions. Finally, it can be concluded that the additional expense of the texture measurement, calculation of $\mathrm{C}_{\mathrm{ijk}}$ and determination of four case-specific calibration functions is worth the effort for the application of the incremental hole drilling method for strongly textured material states as in the present case the deep drawn DP600-steel cup.

\section{Acknowledgement}

The Graduate School 1483 "Process chains in production: Modelling, interactions and assessments of process zones" and the German research foundation (DFG) are gratefully acknowledged for funding. Further, we thank Prof. M. Liewald, P. Schmid and his team at the Institute for Metal Forming Technology in Stuttgart for the realization and support during the deep drawing experiments.

\section{References}

[1] E. Macherauch, P. Müller, Das $\sin ^{2} \psi$-Verfahren der röntgenographischen Spannungsmessung, $\mathrm{Z}$ angew. Physik, 13 (1961) 340-345.

[2] V. Hauk, G. Vaessen, Eigenspannungen in Kristallitgruppen texturierter Stähle, Z. Metallkde., 76 (1985) 102-107.

[3] H. Behnken, V. Hauk, Berechnung der röntgenographischen Spannungsfaktoren texturierter Werkstoffe - Vergleich mit experimentellen Ergebnissen, Z. Metallkde., 82 (1991) 151-158.

[4] G. S. Schajer, Measurement of non-uniform residual stresses using the hole-drilling method. Part I - Stress calculation procedures, J. Eng. Mater. Technol., 110 (1988) 338-343. http://dx.doi.org/10.1115/1.3226059

[5] T. Schwarz, H. Kockelmann, Die Bohrlochmethode - ein für viele Anwendungsbereiche optimales Verfahren zur experimentellen Ermittlung von Eigenspannungen, Messtech. Briefe, 29 (1993) 33-38.

[6] S. Schuster, J. Gibmeier, Residual stress analysis of strongly textured materials by means of the incremental hole-drilling method - Survey on the application limits, Mater. Test., 56 (2014) 915-922. http://dx.doi.org/10.3139/120.110651

[7] S. Schuster, J. Gibmeier, Incremental hole-drilling for residual stress analysis of strongly textured material states - A new calibration approach, Exp. Mech., 56 (2016) 369-380. http://dx.doi.org/10.1007/s11340-015-0104-3

[8] R. Hill, The elastic behaviour of a crystalline aggregate, Proc. Phys. Soc. A, 65 (1952) 349354. http://dx.doi.org/10.1088/0370-1298/65/5/307

[9] Landolt-Börnstein, Group III Condensed Matter, Table 3, Cubic system. Elements, 29a:14, Springer, 1992.

[10] R. Hill, A theory of the yielding and plastic flow of anisotropic metals, Proc. R. Soc. Lond. A, 193 (1948) 281-297. http://dx.doi.org/10.1098/rspa.1948.0045 